\title{
Formation of chondrule analogs aboard the International Space Station
}

\author{
Tamara E. KOCH (D) ${ }^{* 1}$, Dominik SPAHR ${ }^{1}$, Beverley J. TKALCEC (D) ${ }^{1}$, Miles LINDNER ${ }^{1}$, \\ David MERGES $^{1}$, Fabian WILDE ${ }^{2}$, Björn WINKLER ${ }^{1}$, and Frank E. BRENKER ${ }^{1,3}$ \\ ${ }^{1}$ Insitute of Geosciences, Goethe University Frankfurt, 60438 Frankfurt am Main, Germany \\ ${ }^{2}$ Helmholtz-Zentrum Hereon, Max-Planck Strasse 1, 21502 Geesthacht, Germany \\ ${ }^{3}$ Hawai'i Institute of Geophysics and Planetology, School of Ocean and Earth Science and Technology, \\ University of Hawai'i at Mānoa, 1680 East-West Road, Honolulu, Hawai'i 96822, USA \\ *Corresponding author. E-mail: t.koch@em.uni-frankfurt.de
}

(Received 16 April 2021; revision accepted 23 July 2021)

\begin{abstract}
Chondrules are thought to play a crucial role in planet formation, but the mechanisms leading to their formation are still a matter of unresolved discussion. So far, experiments designed to understand chondrule formation conditions have been carried out only under the influence of terrestrial gravity. In order to introduce more realistic conditions, we developed a chondrule formation experiment, which was carried out at longterm microgravity aboard the International Space Station. In this experiment, freely levitating forsterite $\left(\mathrm{Mg}_{2} \mathrm{SiO}_{4}\right)$ dust particles were exposed to electric arc discharges, thus simulating chondrule formation via nebular lightning. The arc discharges were able to melt single dust particles completely, which then crystallized with very high cooling rates of $>10^{5} \mathrm{~K} \mathrm{~h}^{-1}$. The crystals in the spherules show a crystallographic preferred orientation of the [010] axes perpendicular to the spherule surface, similar to the preferred orientation observed in some natural chondrules. This microstructure is probably the result of crystallization under microgravity conditions. Furthermore, the spherules interacted with the surrounding gas during crystallization. We show that this type of experiment is able to form spherules, which show some similarities with the morphology of chondrules despite very short heating pulses and high cooling rates.
\end{abstract}

\section{INTRODUCTION}

The formation of chondrules is considered to be a fundamental step in the evolution of our solar system and chondrules are often termed as the building blocks of the terrestrial planets (Johansen et al. 2015; Bollard et al. 2017). Besides their important role in planet formation, they contain information about their precursor material and the conditions and dynamics of the early protoplanetary disk (e.g., Tenner et al. 2013, 2015; Ushikubo et al. 2013; Schrader et al. 2018, 2020; Marrocchi et al. 2019). However, the formation process of chondrules remains enigmatic despite numerous proposed formation theories (e.g., reviewed in Boss 1996; Desch et al. 2012; Connolly and Jones 2016; Russell et al. 2018). The challenge lies in developing a model in which the morphological, structural, and chemical properties observed in chondrules result from a physically plausible formation mechanism (reviews in Desch and Cuzzi 2000; Desch et al. 2012; Hubbard and Ebel 2018). Several chondrule formation scenarios have been proposed, such as shock waves (e.g., Ciesla and Hood 2002; Morris and Boley 2018), collisions of planetesimals (Krot et al. 2005; Sanders and Scott 2012; Lichtenberg et al. 2018), dissipation of magneto hydrodynamic turbulence (McNally et al. 2013), or nebular lightning (Horányi et al. 1995; Desch and Cuzzi 2000), whereby some are considered more plausible than others (Desch et al. 2012). However, none of these theories is able to explain all features typical for a specific chondrule type by a single formation mechanism.

Experiments have been undertaken to test whether specific formation conditions lead to the formation of chondrules with a set of properties similar to those observed in natural samples, for example, the different chondrule textures and chemical zoning in olivine 

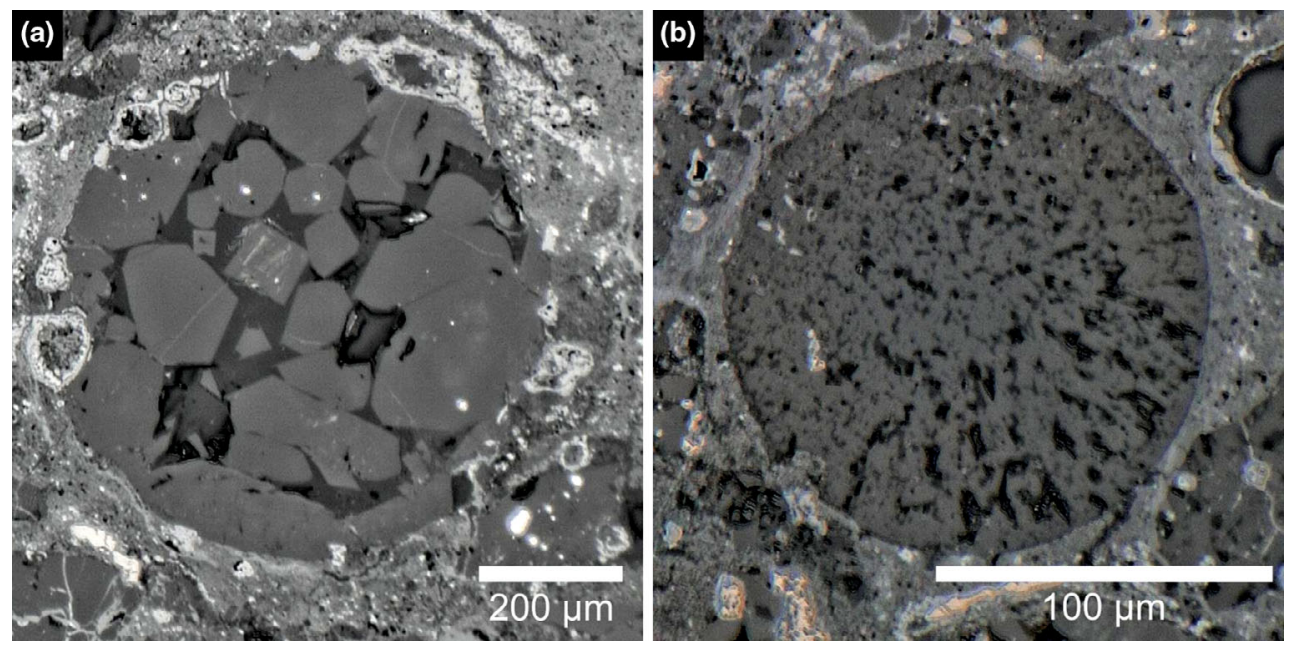

Fig. 1. Light microscopic images of different chondrule types from Northwest Africa 11542 (Gattacceca et al. 2019), which were probably formed under different thermal conditions. a) A porphyritic olivine chondrule consisting of olivine crystals in mesostasis. b) A cryptocrystalline chondrule. (Color figure can be viewed at wileyonlinelibrary.com.)

crystals (e.g., Connolly et al. 1998; Hewins and Fox 2004; Hewins et al. 2005; Jones et al. 2005, 2018; Villeneuve et al. 2015). One of the key factors involved in such experiments is the choice of the heat source. Furnace heating experiments, for example, have so far been significantly more successful in producing chondrule-like characteristics compared to flash heating experiments (Wdowiak 1983; Hewins et al. 2000; Güttler et al. 2008; Poppe et al. 2015) and have provided some constraints on the thermal evolution of chondrules. For example, porphyritic chondrules, which represent the most abundant chondrule type (e.g., Russell et al. 2018), are considered to be the result of incomplete melting followed by slow cooling at rates of 50-500 $\mathrm{K} \mathrm{h}^{-1}$ (Jones et al. [2018] and references therein), whereas cryptocrystalline or radial chondrule textures are thought to be the result of heating above the liquidus and subsequent fast cooling with approximately $1000 \mathrm{~K} \mathrm{~h}^{-1}$ (Jones et al. 2018). Figure 1 shows a representative porphyritic chondrule and a cryptocrystalline chondrule; the different textures possibly result from different thermal histories.

Furthermore, interactions between the chondrule precursor material with the surrounding gas during chondrule formation are considered to be relevant for chondrule formation (Tissandier et al. 2002; Libourel et al. 2006; Marrocchi and Libourel 2013; Marrocchi and Chaussidon 2015; Villeneuve et al. 2015; Piani et al. 2016; Chaumard et al. 2018; Marrocchi et al. 2018, 2019; Libourel and Portail 2018). Experiments on gas-melt interactions have successfully reproduced different chondrule characteristics pointing toward the need to go beyond models based on crystallization within closed systems (Tissandier et al. 2002; Villeneuve et al. 2015).

Yet, these experiments were all unavoidably influenced by the Earth's gravity. In consequence, more realistic chondrule formation experiments must involve long-term microgravity conditions, which are offered today only aboard the International Space Station (ISS). This environment brings several benefits. First, the sample material does not have to be statically mounted and single dust particles can serve as sample material instead of pressed pellets. Second, in microgravity, the thermal evolution of the heated samples is not influenced by thermally induced convection or prolonged contact of the sample with a container wall or sample holder. Furthermore, crystallization at microgravity probably avoids gravitationally induced settling of crystals on one side of the sample (Radomsky and Hewins 1990). Instead, the heated samples can cool and crystallize undisturbed while floating freely, similar to melt spherules in the solar nebula.

Although the theory of chondrule formation by lightning in the solar nebula has not been central to the debate in the last decade, there are many insights that can be gained from an experiment that uses electrical discharges as heat source. First, chondrule formation by lightning can explain several chondrule characteristics, such as the chondrule matrix complementarity paired with highly variable thermal histories of adjacent chondrules (Boss 1996; Desch et al. 2012; Connolly and Jones 2016). A recent study addressed the mechanism leading to lightning, and proposed the decay of ${ }^{26} \mathrm{Al}$ as a plausible charging mechanism (Johansen and Okuzumi 2018), thus affirming that nebular lightning 
can provide the required amount of energy to form the estimated mass of chondrules of at least $10^{24} \mathrm{~g}$ (Levy 1988; Desch and Cuzzi 2000). Studies focusing on gasmelt interactions have questioned whether the formation of porphyritic chondrules requires slow cooling rates, and proposed faster cooling rates, which would be consistent with the cooling rate of chondrules being heated by an arc discharge (Libourel and Portail 2018). The chemical zoning of $\mathrm{Fe}, \mathrm{Ca}$, and $\mathrm{Cr}$ observed in chondrule olivine has been presented as an argument for slow cooling (Jones 1990; Hewins et al. 2005; Jones et al. 2018), but recent experiments have produced porphyritic textures with $\mathrm{Al}$ and $\mathrm{Ti}$ zoning in olivines, similar to natural chondrules, with high cooling rates of 3000-6000 K h${ }^{-1}$ (Greenwood and Herbst 2021). This distribution is similar to $\mathrm{Al}-\mathrm{Ti}$ zoning reported in the literature (Libourel and Portail 2018; Marrocchi et al. 2018, 2019), thus substantially weakening the argument that slow cooling is required for the formation of porphyric chondrules.

Access to new commercially available payloads aboard the ISS enabled the development of a chondrule formation experiment at long-term microgravity with the acronym EXCISS (experimental chondrule formation aboard the ISS; Spahr et al. 2020). The experimental setup was designed to fit in a $10 \times 10 \times 15 \mathrm{~cm}^{3}$-sized experiment container and adhere to the strict constraints regarding technical components and power supply (Spahr et al. 2020). As this type of experiment was to be performed at the ISS for the first time, the experimental conditions had to be as simple as possible but still reflect a realistic model system. Therefore, in this experiment, freely floating $\mathrm{Mg}_{2} \mathrm{SiO}_{4}$ dust particles were exposed to arc discharges inside a glass sample chamber. In the past, experiments using lightning or similar flash heating mechanisms (e.g., laser or plasma arcs) have not been very successful, either leading to the destruction of the samples or failing to reproduce chondrule textures (Blander et al. 1976; Güttler et al. 2008; Morlok et al. 2012; Poppe et al. 2015). However, arc discharge properties such as size, duration, and discharge energy can be customized and we were able to modify the arc discharge generation technique in our experiment (Spahr et al. 2020) to produce less energetic, longer arc discharges compared to previous experiments (Güttler et al. 2008). A camera allowed the observation of the particles in real time and the sample material could be analyzed after sample return.

The proof-of-concept experiment presented here shows that scientifically useful results regarding chondrule formation, such as the response of dust particles to arc discharges, crystallization under microgravity conditions, and the subsequent thermal evolution of chondrules, can be obtained under very tight technical constraints and we present the analysis of melt spherules formed in microgravity and discuss their relevance as chondrule analogs.

\section{METHODS AND MATERIALS}

\section{ISS Experiments}

The experimental setup and the calibration of the arc discharges are described in Spahr et al. (2020). The experimental setup was mounted inside a $10 \times 10 \times 15 \mathrm{~cm}^{3}$ sized NanoRacks NanoLab, which was connected to the ISS by USB. The experiment was installed in the NanoRacks platform and the total run time of the experiment was 30 days. In total, 81 arc discharges with energies of 5-8 $\mathrm{J}$ and durations between 300 and $500 \mu$ s per discharge were induced. The sample chamber was manufactured from quartz glass and two tungsten electrodes with a diameter of $1 \mathrm{~mm}$ were fused into the glass on opposite sides. The sample chamber was filled with sample material and Argon atmosphere with 100(1) mbar. The complete sample material remained in the sample chamber during the experiments. These experiments were filmed and the video material could be downloaded on a daily basis. Due to the low frame rate caused by the experimental setup requirements, it is not possible to distinguish whether the particles melted inside the plasma channel or by thermal radiation.

\section{Analytical Procedure and Sample Preparation}

Scanning electron microscopy (SEM) imaging was performed using a Phenom World ProX desktop SEM equipped with an electron backscatter detector (BSE) at the Geoscience Institute at the Goethe University. Energy-dispersive X-ray spectroscopy (EDX) was carried out with an integrated silicon drift detector for a semiquantitative characterization of the chemical composition. The non-coated samples were measured under low vacuum conditions; the polished and carboncoated samples were measured under high vacuum conditions. For imaging, we used an acceleration voltage of $10 \mathrm{kV}$ while for EDX analysis, a voltage of $15 \mathrm{kV}$ was employed. Multiple BSE images were acquired with different focal planes and combined afterward with the Helicon Focus 7 Pro software from Helicon. EDX data were analyzed using the Phenom Pro Suite software.

EBSD fabric analysis is especially suited to identify structural and textural properties of materials and is increasingly employed as an analytic tool to investigate the remnants of solar nebular processes in planetary materials (Bland et al. 2011; Tkalcec et al. 2013). EBSD was performed at the Geoscience Institute at Goethe 
University Frankfurt with a Jeol scanning electron microscope JSM 6490. The SEM is equipped with a Nordlys II phosphor screen EBSD detector with Channel 5 software from Oxford Instruments and HKL Technology. EBSD was performed with an acceleration voltage of $15 \mathrm{kV}$, a working distance of $20 \mathrm{~mm}$, and a Siwafer as calibrant. EBSD data were acquired by automated mapping performed at step sizes of $0.8-1 \mu \mathrm{m}$ with low gain, $2 \times 2$ pixel binning, and a mean angular deviation limit of $<1.3^{\circ}$. Following data acquisition, no noise reduction at all was performed on the acquired EBSD data. Contoured pole figures are displayed as multiples of uniform density, with a half width of $10^{\circ}$. The initial sample material as well as the experimental outcomes were embedded in Araldite epoxy resin, polished with Syton polish, and thinly coated with carbon.

Synchrotron-based micro-CT was performed at PETRA III (DESY) in Hamburg, Germany. All data were collected on the microtomography beamline P05 (Ogurreck et al. 2013; Moosmann et al. 2014; Wilde et al. 2016), operated by Helmholtz-Zentrum Hereon, using a $15 \mathrm{keV}$ beam monochromatized by a double crystal monochromator. The images were acquired with a $24 \times$ magnification using a CMOS camera resulting in an approximately $1.8 \times 1.8 \mathrm{~mm}^{2}$ field of view. We used an acquisition time of $350 \mathrm{~ms}$. A binning factor of $2 \times 2$ pixel resulted in a binned pixel size of approximately $0.61 \times 0.61 \mu^{2}$. The samples were completely embedded in Araldite epoxy resin.

\section{Initial Sample Material}

The initial sample material consisted of wellcharacterized synthetic forsterite particles (Spahr et al. 2020). Figure $2 \mathrm{a}$ shows a focus-stacked SEM BSE image of a representative initial particle. Figure $2 b$ shows several embedded and polished initial grains. The particles include numerous, evenly distributed, small pores. The grains are irregularly shaped with rough surfaces. A grain size analysis of the initial sample material was carried out with a random sampling of 849 grains using ImageJ (Rueden et al. 2017). Figure 2c shows a histogram of the longest dimension of the grains fitted with a normal distribution function. The mean diameter obtained from the fit is $126 \mu \mathrm{m}$ and, due to the heterogeneity of the particle shapes, the distribution is rather broad $(\sigma=23)$. Figure $2 \mathrm{~d}$ shows the correlation of the major to the minor ellipse axes, which again indicates the variability of the particle morphologies. EBSD analysis of the initial particles showed that the particles are fine grained, consisting of crystals having (circular equivalent) diameters of 5$10 \mu \mathrm{m}$, with a few exceptions where larger crystals
$>20 \mu \mathrm{m}$ are present (Figs. 3a and 3b). Within each grain, the crystals are randomly oriented (Fig. 3c).

The sample chamber was loaded with $30 \mathrm{mg}$ of the sample material. Approximating the volume of a single grain by a sphere with a diameter of $126 \mu \mathrm{m}$ and a porosity of approximately $10 \mathrm{vol} \%$, the total number of particles is in the range of $10^{4}$ particles.

\section{Cooling Rate Determination}

The cooling rates of chondrules melted by lightning are usually modeled by radiative cooling of a gray body (Equation 1; Morris and Desch 2010). The temperature of the chondrule analog after the arc discharge is given by the liquidus temperature of forsterite of $2163 \mathrm{~K}$ (Bowen and Schairer 1925) and the temperature aboard the ISS is estimated to be $290 \mathrm{~K}$. Equation 1 gives the change of the internal energy $U$ of a radiating spherical gray body with time:

$$
\frac{\partial U}{\partial t}=\varepsilon \cdot \sigma \cdot A \cdot\left(T_{\text {sample } 0}^{4}-T_{\mathrm{ISS}}^{4}\right),
$$

where $U$ is the internal energy, $t$ is the time after the heating pulse in $s, \epsilon$ is the emissivity (for reference, a black body has an emissivity of 1), $\sigma$ is the StefanBoltzmann constant, $A$ is the surface area of the spherule, and $T_{\text {sample }}$ is the temperature of the heated spherule at time 0 and $T_{I S S}$ is the temperature of the surrounding, both in $\mathrm{K} . \Delta U$ for a short $d t$ can be obtained using Equation 2.

$$
\Delta U=\varepsilon \cdot \sigma \cdot A \cdot\left(T_{\text {sample }}^{4}-T_{\text {ISS }}^{4}\right) \cdot d t .
$$

The change in $U$ can also be described by means of the temperature-dependent specific heat capacity $c_{p}(T)$ in $\mathrm{J} \mathrm{mol}^{-1} \mathrm{~K}^{-1}$, the change in temperature of the spherule $\Delta T$ in $\mathrm{K}$, and the amount of substance in mol (Equation 3). The amount of substance $n$ is calculated for different spherule sizes using the volume of the spherule, the density of forsterite $\left(3.275 \mathrm{~g} \mathrm{~cm}^{-3}\right)$, and the molar mass of forsterite $\left(140.693 \mathrm{~g} \mathrm{~mol}^{-1}\right)$.

$$
U=c_{p}(T) \cdot n \cdot(\Delta T) .
$$

Combining Equations 2 and 3 gives an expression for the change of temperature of the spherule during a short time of radiation (Equation 4).

$$
\Delta T=\frac{\varepsilon \cdot \sigma \cdot A \cdot\left(T_{\text {sample } 0}^{4}-T_{\mathrm{ISS}}^{4}\right) \cdot d t}{c_{p}(T) \cdot n} .
$$



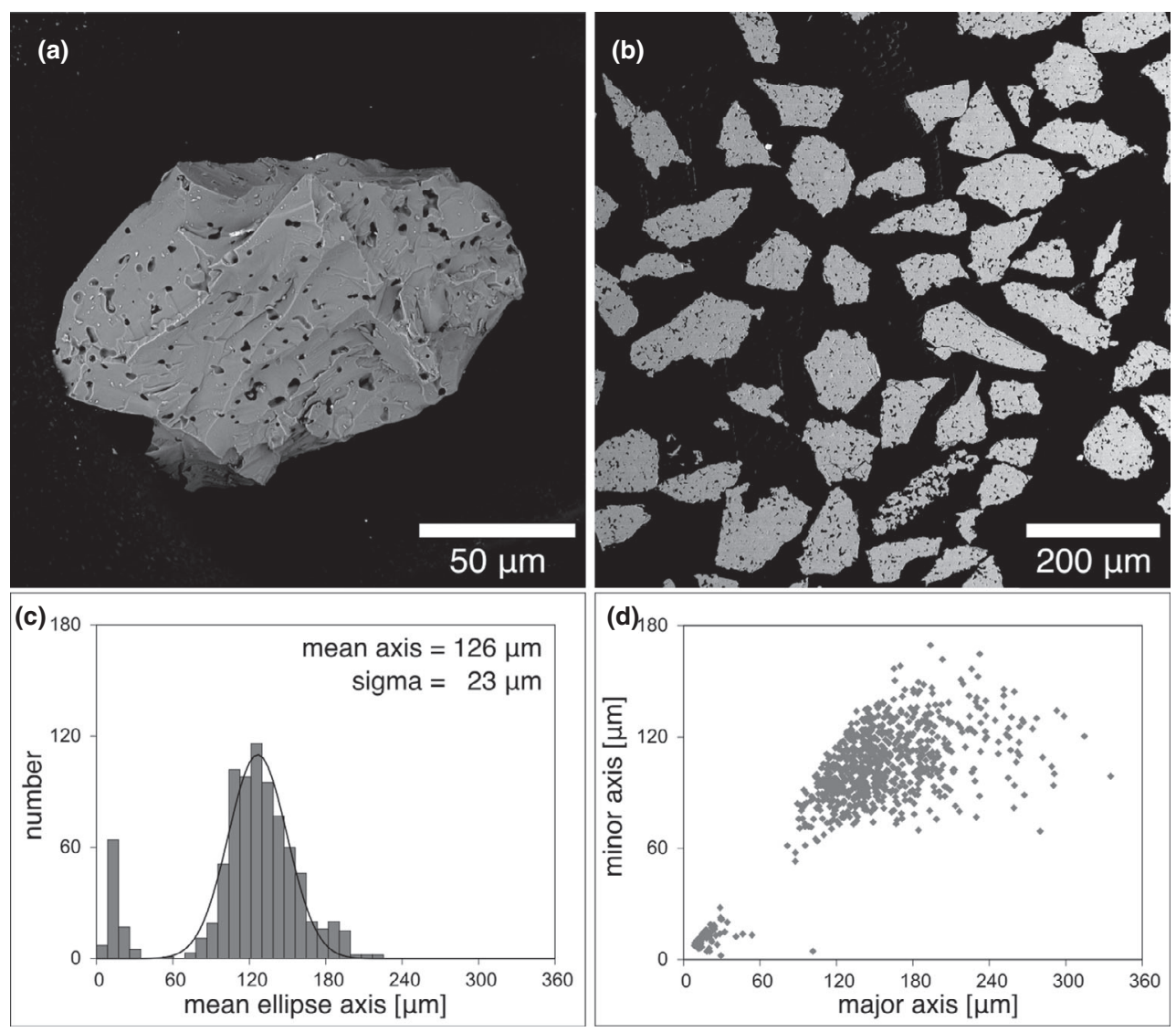

Fig. 2. Initial sample material. a) Focus-stacked representative porous initial dust particle used as starting material. b) Polished grains of the initial starting material. The particles have highly heterogeneous morphologies and a large amount of evenly distributed small pores. c) Histogram of the grain size distribution of a random sample containing 849 grains fitted with a normal distribution function. d) Correlation between the minor and major axes of best-fit ellipses of the initial material.

The resulting $\Delta T$ is subtracted from $T_{\text {sample } 0}$ to derive the new temperature of the spherule $T_{\text {samplel }}$. Using this new temperature, the calculations are iterated $N$ times until $\mathrm{T}_{\text {sample } N}$ is sufficiently close to $T_{\text {ISS }}(291 \mathrm{~K}$ in this case). The specific heat capacity $c_{p}(T)$ in Equation 3 is calculated for each iteration step with the data of the NIST Standard Reference Database (see National Institute of Standards and Technology [NIST n.d.] in the reference section).

\section{RESULTS}

\section{Video Analysis of the Experiments}

The videos and photographs downloaded from the ISS show that the first few arc discharges were induced while the space between the electrodes was empty. Then, when an electric field was applied prior to the next discharge, the particles agitated by the previous arc discharges formed an aggregate levitating between the electrodes (Fig. 4a). This aggregate was hit directly once by the ensuing arc discharge. The number of particles in the aggregates between the electrodes each time decreased strongly with ongoing arc discharges until their presence became an infrequent occurrence. In all, the space between the electrodes was empty in $70 \%$ of the arc discharges. In total, 81 arc discharges were induced. The first noticeable change in particle morphology is observed after the fourth arc discharge. The number of transformed particles observed in the field of view increased with ongoing experimental duration. However, the actual process of transformation of individual particles could not be observed. Figure $4 \mathrm{~b}$ shows an example of a melt spherule at a late stage of the experiment that may be a chondrule analog.

\section{SEM and CT Analysis}

After the return of the sample chamber to the Earth, the melt spherules formed during the experiment were studied with SEM combined with EBSD and synchrotron micro-CT. More than 90 newly formed objects were isolated from the sample chamber. SEM and synchrotron micro-CT on these objects show nearly 

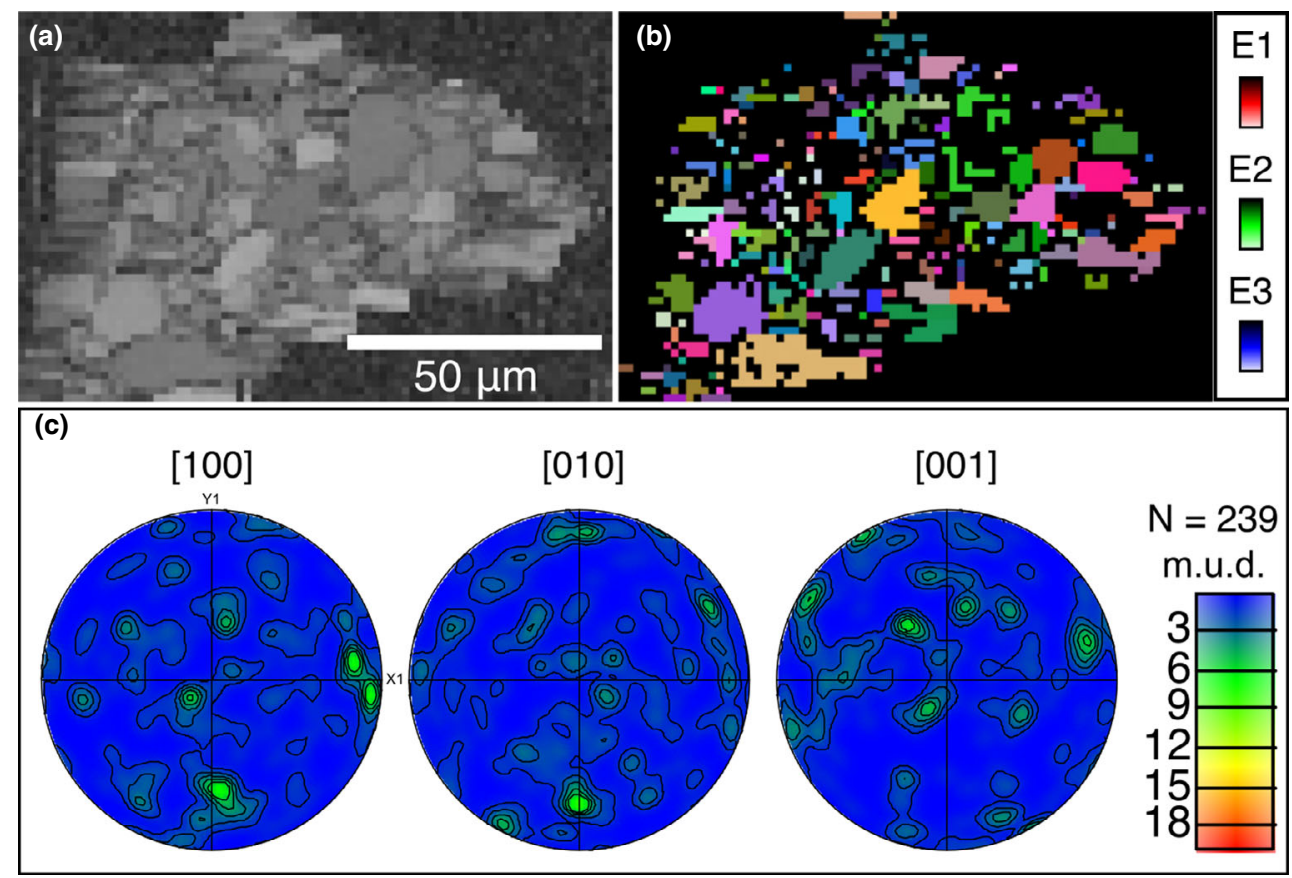

Fig. 3. EBSD analysis of one typical polished initial sample grain from Fig. 1b. a) Band contrast image. The particles are fine grained with most crystals having (circular equivalent) diameters of 5-10 $\mu \mathrm{m}$ with a few exceptions $>20 \mu \mathrm{m}$. b) All-Euler map of forsterite. c) Pole figures showing the contoured data (bottom) based on one point per grain, identifying 239 grains. The pole figures reveal a random orientation. Since no noise reduction was applied to the raw data, the few tight point maxima (green) are likely caused by duplication (non-adjacent points measured from the same grain but identified as individual grains) in areas where indexing was not continuous. (Color figure can be viewed at wileyonlinelibrary.com.)
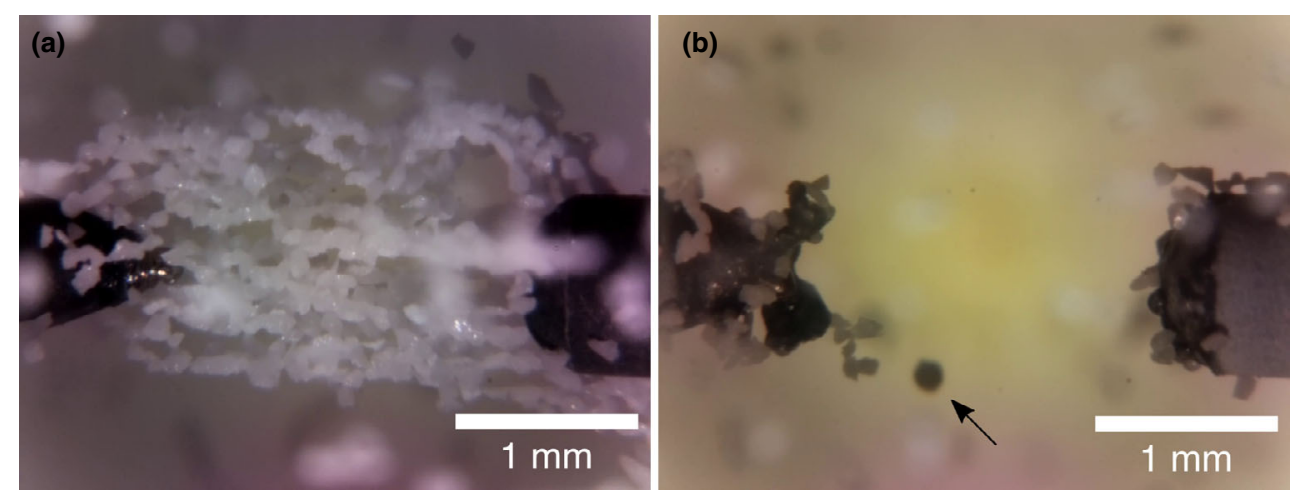

Fig. 4. Field of view of the sample chamber of the experiments aboard the ISS. a) An aggregate of particles levitating between the electrodes prior to an arc discharge. b) A melt spherule formed by arc discharge, levitating inside the sample chamber at a late stage of the experiments (black arrow). (Color figure can be viewed at wileyonlinelibrary.com.)

perfect, spherical shapes with smooth surfaces, very distinct from the rough, porous, angular, and irregular shapes of the initial particles (Figs. 5a-c). SEM, EDX, and optical observations show that tungsten, most likely sputtered from the electrodes, has interacted with the forsteritic melt and appears as bright lines on the spherule surface. During cooling and crystallization of forsterite, tungsten has precipitated along grain and subgrain boundaries, thus allowing a clear identification of the grain boundaries on the surface of the spherules (Figs. 5a-c). Micro-CT projections also showed the smooth surface on the spherical objects (Fig. 5d).

Cross sections of some typical spherules were subsequently analyzed regarding their microstructure with SEM (Fig. 6). The melt spherules are nearly perfectly round with diameters ranging from 75 to $100 \mu \mathrm{m}$ in size. The initial pore structure observed in the starting grains (Figs. 2a and 2b) has disappeared 

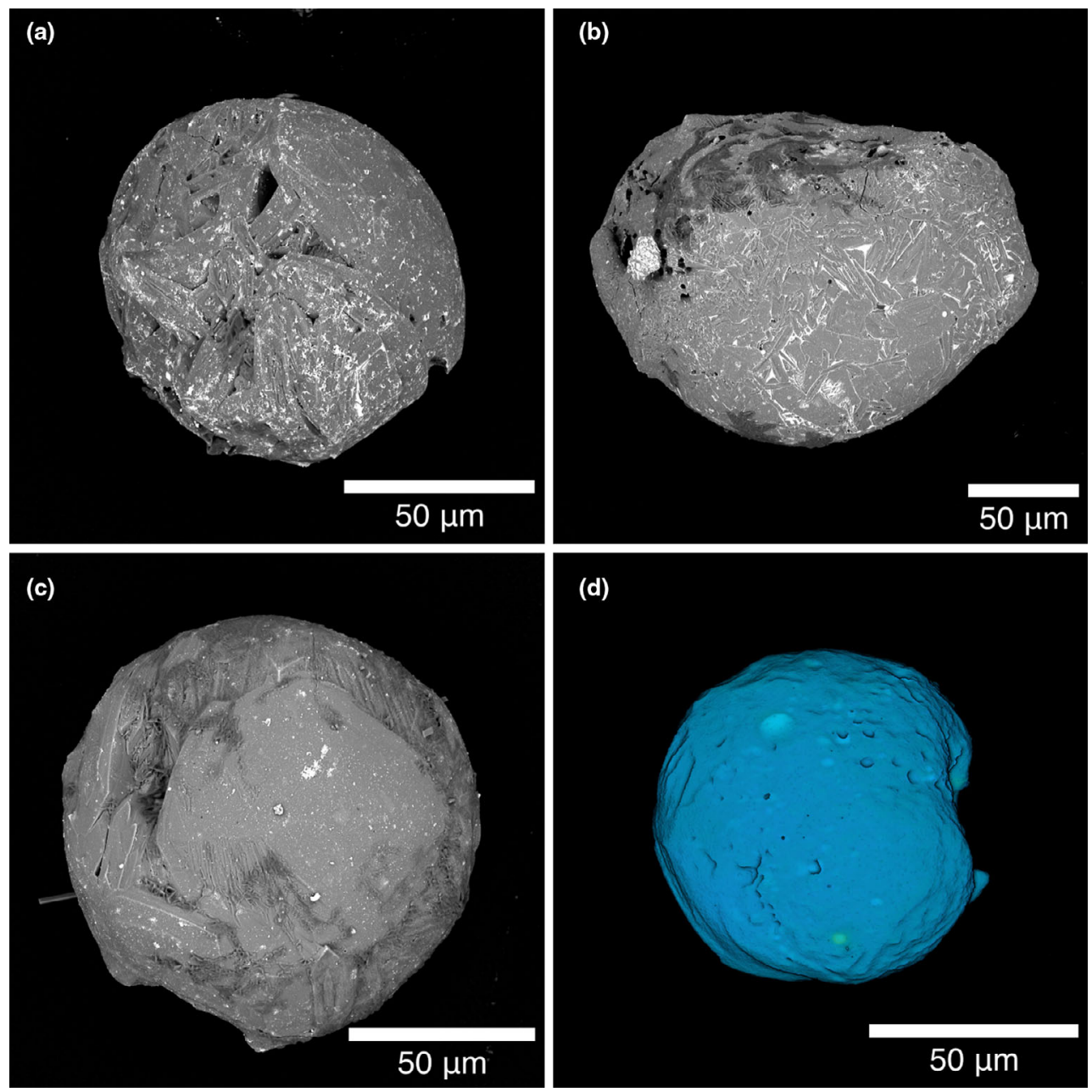

Fig. 5. SEM BSE images and micro-CT projection of some exemplary melt spherules. a-c) Focus-stacked BSE images of melt spherules. The shape and surface structure are completely transformed compared to the initial grains. The single grains are clearly visible. d) Projections of a melt spherule obtained by synchrotron micro-CT. (Color figure can be viewed at wileyonlinelibrary.com.)

completely and instead a few larger voids have formed (Figs. 6a-d). Tungsten precipitations form a network of fine lines appearing bright in the BSE images and are often arranged as bundles of parallel lamellae. In addition to the individual melt spherules, the experiments produced objects consisting of several fused spherules. Figure $5 \mathrm{~d}$ shows a BSE image of a cross section of one of these fused objects. Beside melt zones, these aggregates often include still unmolten areas, as can be inferred from the pore structure, which is typical for the pristine material, with smooth interfaces between the molten and unmolten areas. A close up of a molten region shown in Fig. 6e shows the igneous texture with subhedral or elongated grains separated by a network of fine tungsten precipitations and containing a multitude of tiny inclusions (Fig. 6e). An elemental EDX map displaying the distribution of tungsten confirms the tungsten composition of these white grain boundaries, lines, and inclusions (Fig. 6f) and reveals that tungsten is also present in several triple junctions. The amount of tungsten incorporated in each spherule appears to vary from object to object, though this could also be an artifact due to sample preparation.

\section{EBSD Fabric Analysis}

The melt spherules were studied by EBSD with regard to their crystallographic microstructure. Figure 7a shows a BSE image of a polished melt spherule. The band contrast image in Fig. $7 \mathrm{~b}$ reveals a range of crystal sizes between 5 and $20 \mu \mathrm{m}$ and a shapepreferred orientation of most crystals with the longest axes extending parallel to the surface of the spherule. The crystals in areas with a higher tungsten abundance (right-hand side) are larger than those in regions with no visible tungsten precipitations (left-hand side). All 

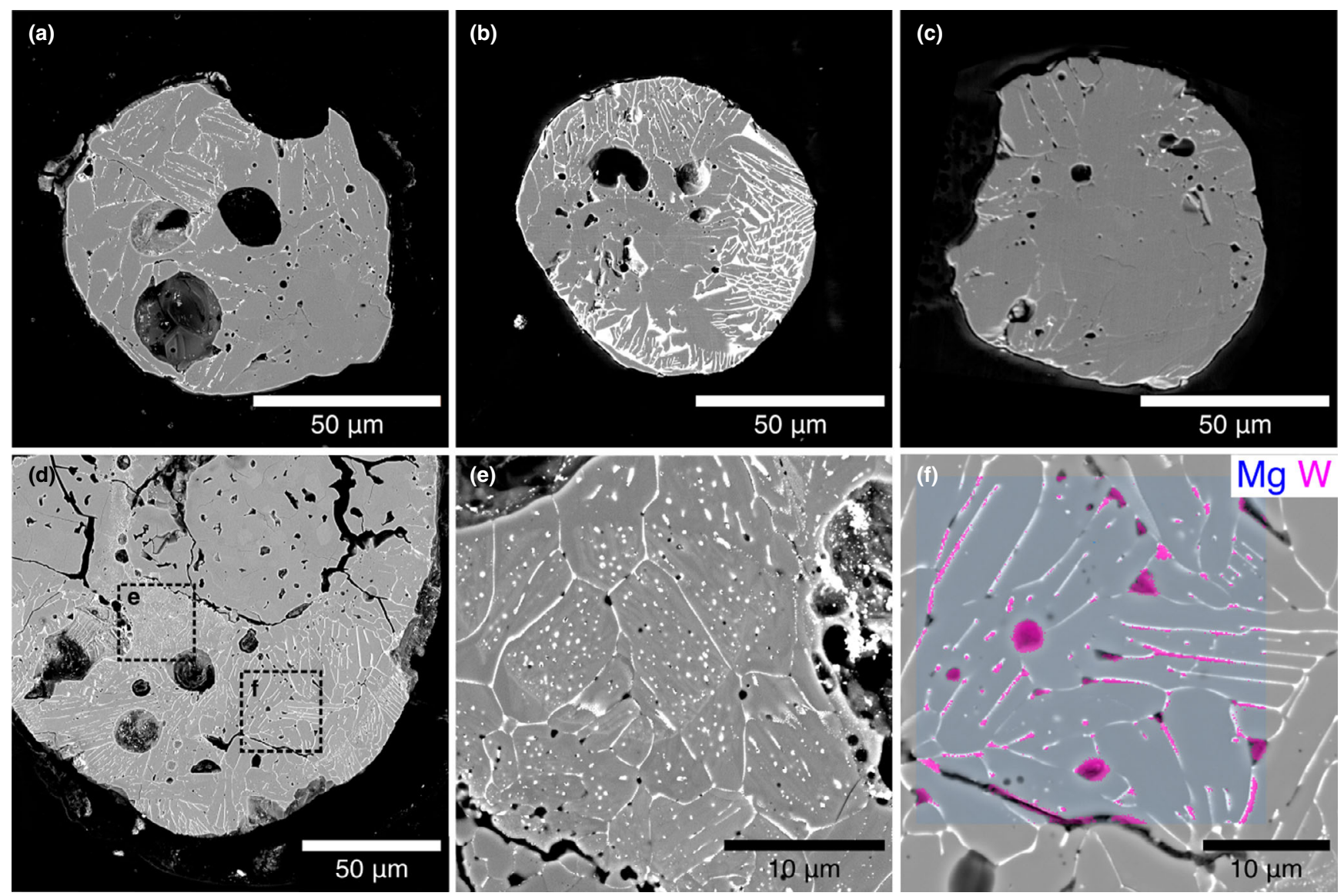

Fig. 6. SEM BSE images of the melt spherules newly formed aboard the ISS. a-c) Polished cross sections of example melt spherules. White lines are tungsten precipitations. Some spherules contain larger voids, which represent gas bubbles replacing the initial pore structure. d) Close-up of an object containing both molten and unmolten areas, the latter showing the initial pore structure of the starting material. The molten area displays tungsten precipitations and larger gas bubbles. e) Close-up of the lefthand square marked in (d). The molten area shows an igneous texture with subhedral forsterite grains exhibiting straight grain boundaries. Tungsten not only precipitates along the grain boundaries but also appears as small tungsten inclusions. f) Close-up of the right-hand square marked in (d). The spatial distribution of $\mathrm{Mg}$ and $\mathrm{W}$ confirm that all bright lines in the BSE image are due to W. (Color figure can be viewed at wileyonlinelibrary.com.)

indexed EBSD patterns were assigned to the forsterite structure. The corresponding all Euler angle map is shown in Fig. 7c. Further, the pole figures reveal a welldefined crystallographic preferred orientation with the [010] axes perpendicular to the sphere surface (Fig. 7), which is completely different from the random orientation of the crystals in the grains of the pristine sample material (Fig. 3).

The fused melt spherules shown in Figs. 6d-f were studied in greater detail. The BSE image in Fig. 8a shows a cross section through the whole object with approximated dimensions of $185 \times 85 \mu^{2}$ in which an area was selected for further EBSD analysis (Fig. 8a, white box). The EBSD band contrast image in Fig. $8 \mathrm{~b}$ shows a noticeable difference in the texture between those parts that have experienced melting and the pristine material. EBSD analysis of an area of the sample, which displays a clear pore structure (righthand area of white box in Figs. 8a and 8c) reveals the microstructure of the initial sample material with forsterite grains in the size range of about $5-10 \mu \mathrm{m}$. This pristine material shows neither shape nor crystallographic preferred orientation (Fig. 8d).

In contrast, EBSD analysis of the area without such pore structure (left-hand area of white box in Figs. 8a and $8 \mathrm{c}$ ) reveals a coarser grain size with forsterite grains averaging approximately $20 \mu \mathrm{m}$. The crystals of this area show a clear crystallographic preferred orientation of the [010] axes (Fig. 8e). The SEM BSE close-up image (Fig. 9a) reveals that the grains displayed in the EBSD phase map (Fig. 9b) include parallel forsterite lamellae separated by tungsten precipitation. The misorientation between these lamellae is negligible $\left(<0.5^{\circ}\right)$ (Fig. 9). 

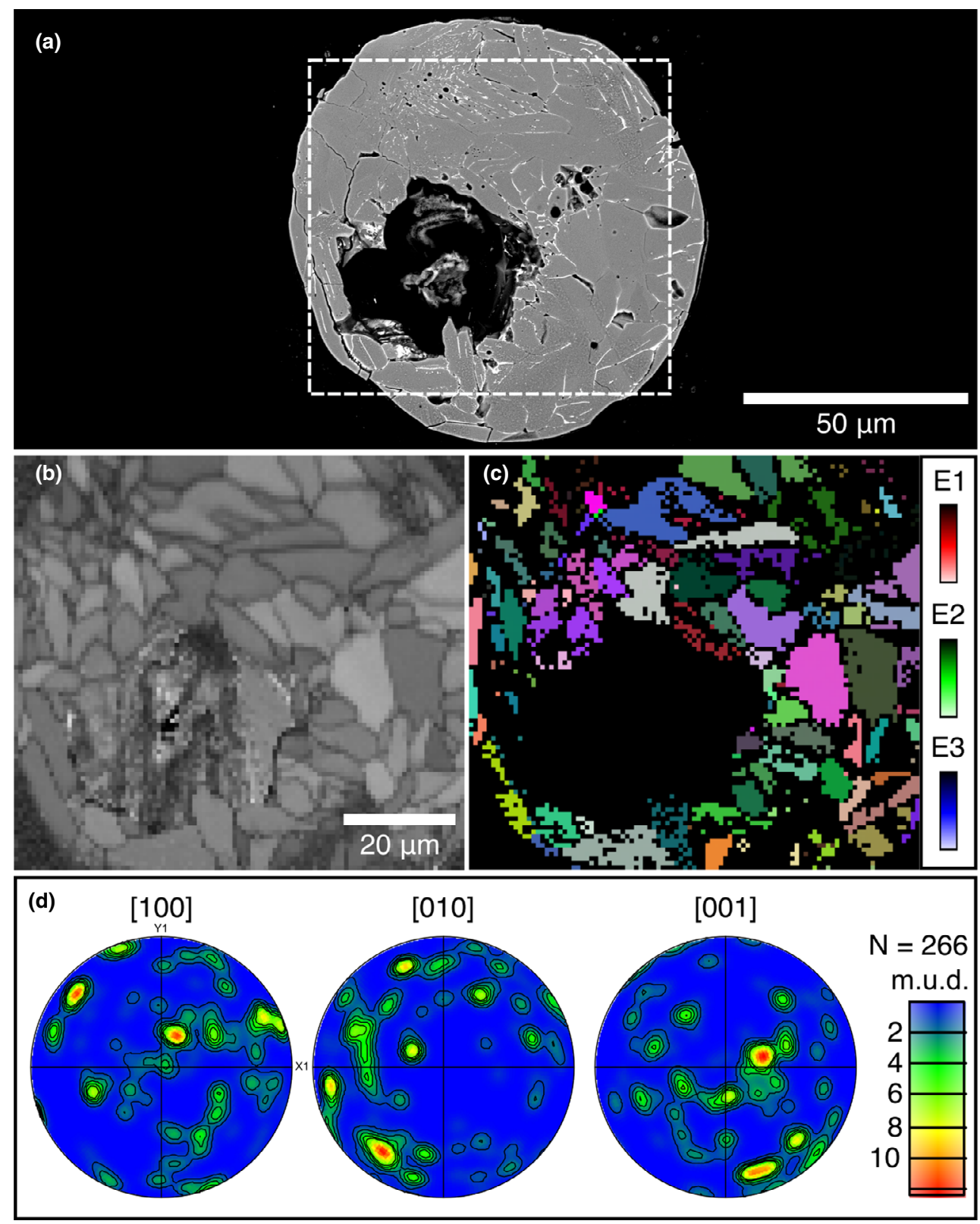

Fig. 7. SEM and EBSD analysis of a representative melt spherule. a) BSE image of the whole melt spherule with a diameter of $100(5) \mu \mathrm{m}$. The dashed square marks the area analyzed by EBSD. b) Grayscale band contrast image visualizing the crystal sizes and morphologies. The grains sizes in the top left, W-poor part of the droplet are slightly smaller than in the right half. c) EBSD all Euler angle map of forsterite. d) Stereographic projections of EBSD data of the melt spherules. The data revealed a welldefined preferred orientation of the [010] axis perpendicular to the sphere surface. (Color figure can be viewed at wileyonlinelibra ry.com.)

\section{Cooling Rates}

The cooling rates were calculated in order to estimate the thermal evolution of the melt spherules after arc discharges. Figure 10a shows the temperature profiles of spherical objects with diameters corresponding to those of the melt spherules formed here $(d=80-200 \mu \mathrm{m})$. The resulting cooling rates are plotted in Fig. 10b starting with a cooling rate of $10^{7} \mathrm{~K} \mathrm{~h}^{-1}$ and decrease to $<10^{4} \mathrm{~K} \mathrm{~h}^{-1}$ within $40 \mathrm{~s}$.

\section{DISCUSSION}

In our experiment, single dust particles in the size range of approximately $100-120 \mu \mathrm{m}$ experienced complete melting by a heating pulse with a duration 

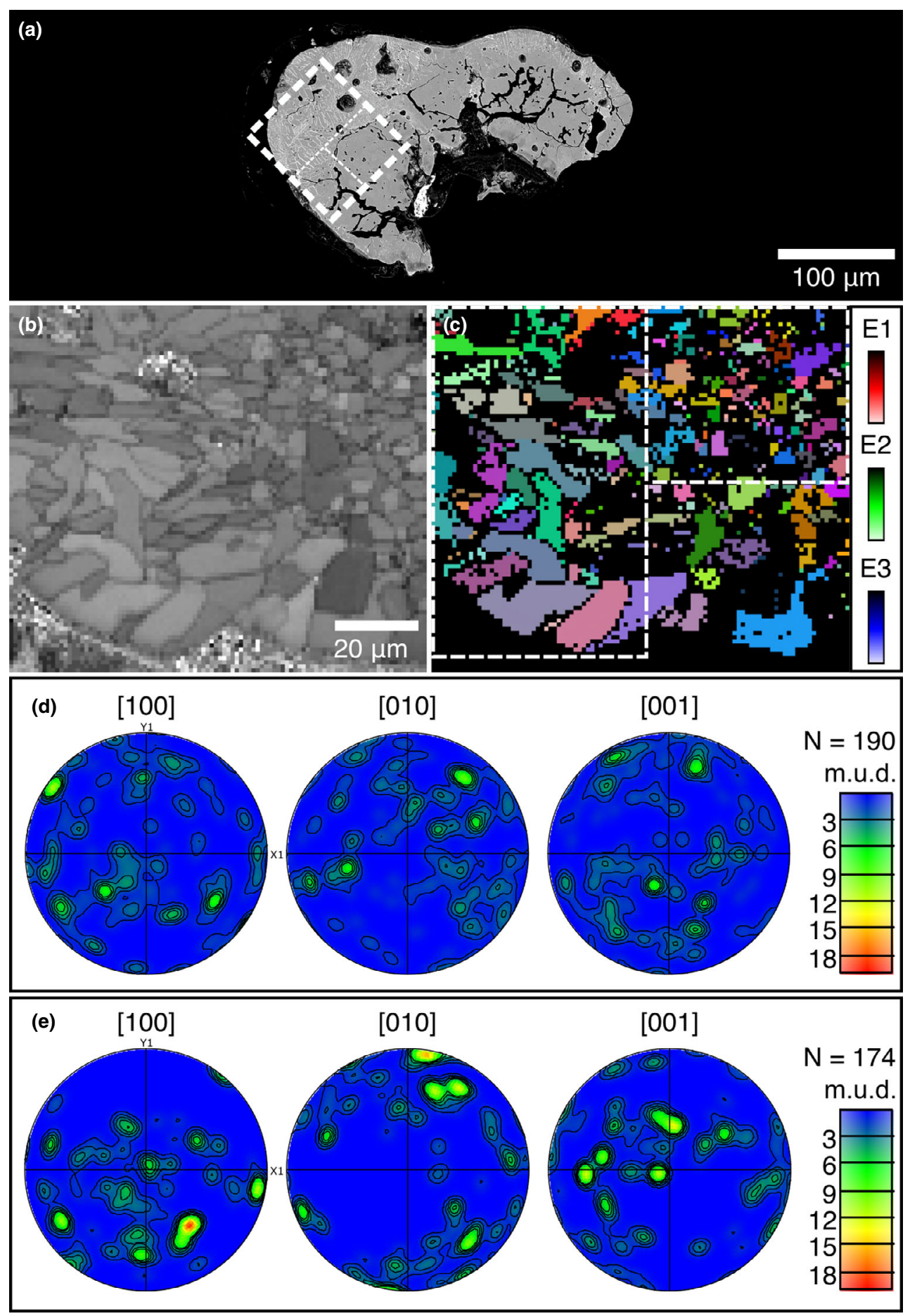

Fig. 8. SEM and EBSD analyses of the object from Fig. 5d. a) BSE image of the fused object consisting of molten and unmolten regions. The dashed square marks the area studied with EBSD. It consists of both an area with the initial pore structure and a molten W-rich area. b) The grayscale band contrast image reveals the microstructure of the studied area. The grain sizes in the left, molten part of the droplet are substantially larger than in the right half that displays the initial pore structure. The crystals close to the rim of the object show an alignment of the longest crystal face perpendicular the surface. c) EBSD all-Euler angle map of forsterite of the area shown in (b). d) Stereographic projections of EBSD data of the initial unmolten part (top right-hand square marked in [c]). e) Stereographic projections of EBSD data of the molten area (left-hand square in [c]) show a similar well-defined alignment of the [010] axes perpendicular to the surface as observed for the melt spherule shown in Fig. 6. (Color figure can be viewed at wileyonlinelibrary.com.) 

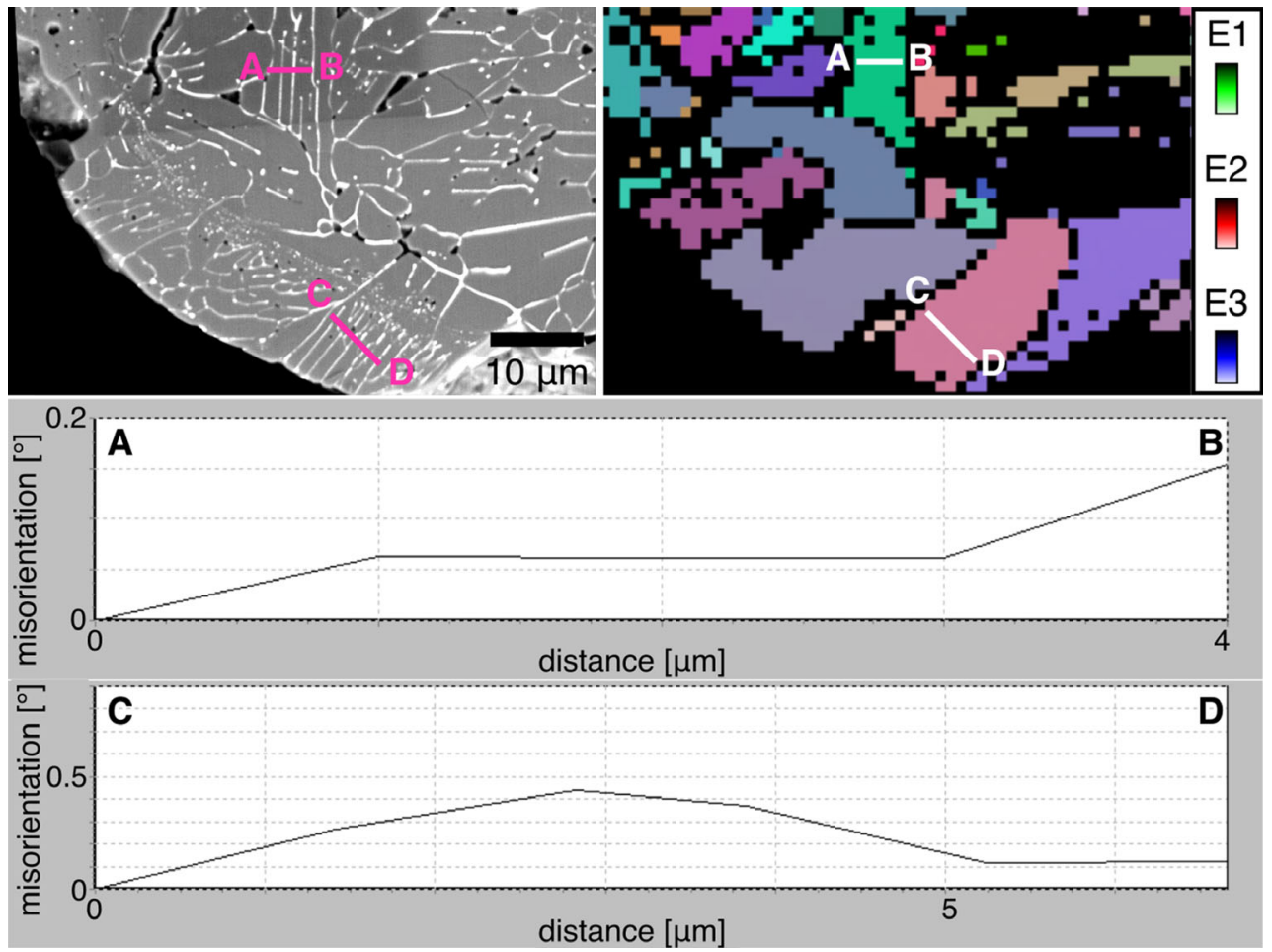

Fig. 9. Misorientation profiles over grains with parallel tungsten lamellae. a) A BSE close-up of the edge of the fused object consisting of molten and unmolten regions. The molten area shows a network of very fine, often parallel tungsten precipitations. b) EBSD all-Euler map displaying the grain boundaries reveals that the observed sets of parallel tungsten lamellae are intracrystal. c) Misorientation profiles perpendicular to the intracrystal tungsten lamella marked in (a) and (b) showing very low misorientation angles $<5^{\circ}$ between the lamella in each set. (Color figure can be viewed at wileyonlinelibrary.com.)

$<500 \mu$ s and energies $<8 \mathrm{~J}$ (Spahr et al. 2020) followed by rapid cooling. The melt spherules have a very narrow size distribution of approximately $80-100 \mu \mathrm{m}$ despite the wider size distribution shown by the initial sample particles. However, the amount of formed spherules is not sufficient for a representative size distribution and for conclusions to be drawn between discharge energy and particle size.

The spherules often include spherical voids, but it is unclear if the voids are due to initial porosity or are the results of inefficient melting, the latter of which was discussed as reason for the formation of voids in previous arc discharge experiments (Wdowiak 1983). In our experiment, the porous starting material allowed us to easily distinguish between molten and unmolten parts but future experiments could benefit from using sample material with no pores in order to determine whether this material develops voids during this type of experiments.

Although the microstructure of the melt spherules obtained here differs from that of natural chondrules, there are some similarities, which merit further discussion and can help to develop the next generation of chondrule formation experiments under microgravity.
The microstructure of these melt spherules is dominated by relatively large forsterite crystals (approximately $20 \mu \mathrm{m}$ ) and few large spherical pores, which is clearly distinct from the microstructure of the initial particles. This implies that the complete particles were melted. Furthermore, crystallization resulted in a strong crystallographic preferred orientation of the [010] axes perpendicular to the spherule surface. This microstructure differs from that of spherules produced in previous flash heating experiments, which mostly display a more radial texture (Blander et al. 1976; Poppe et al. 2015; Mishra et al. 2020). Recent crystallization experiments on Earth using laser heating of $\mathrm{Mg}_{2} \mathrm{SiO}_{4}$ spherules which were levitated on a gas jet led to the formation of a dendritic microstructure, which is clearly different from the fabric observed here (Mishra et al. 2020). Consequently, the observed microstructure seems to be the result of crystallization in microgravity without any contact to sample chamber walls, which would imply that this feature probably cannot be observed in experiments on Earth.

Preferred orientation of [010] has been observed in natural porphyritic and metal-rich porphyritic chondrules (Dodd and Teleky 1967; Jones et al. 2018; 

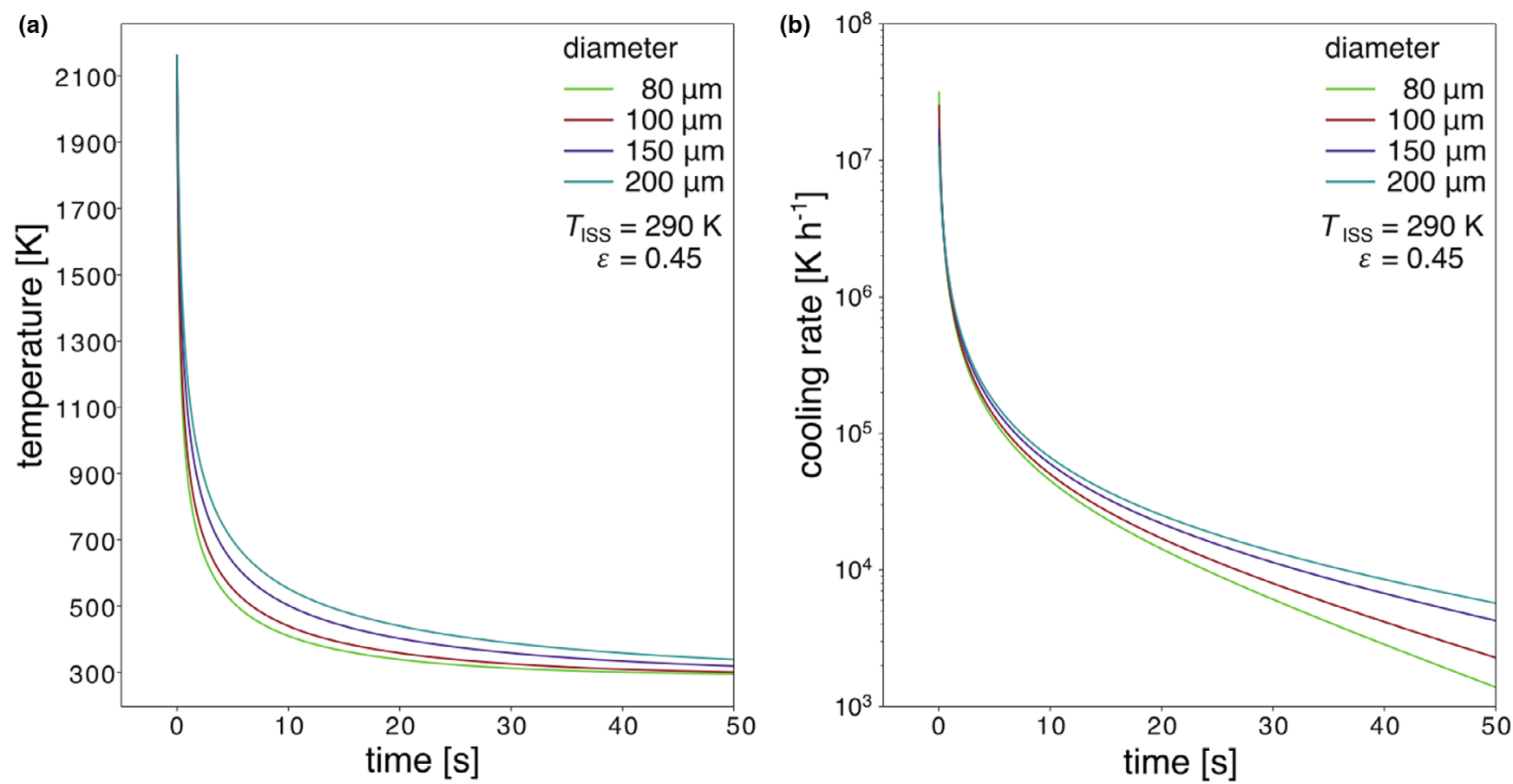

Fig. 10. Calculated cooling histories of the melt spherules after the arc discharge. a) Temperature of the melt spherules plotted against time after arc discharge for different spherule diameters. b) Corresponding cooling rates versus time after heating event. (Color figure can be viewed at wileyonlinelibrary.com.)

Libourel and Portail 2018) and also interpreted as the result of crystallization under microgravity conditions (Dodd and Teleky 1967). The orientations of [100] and [001] of the synthetic samples differ from those of natural chondrules; however, known to the authors, there are no published comprehensive orientation analyses of chondrules required for a detailed comparison.

The molten edge region of one spherule of the fused object in Fig. 8 displays palisadic crystal morphologies which have also been observed in porphyritic chondrules (e.g., Libourel and Portail 2018; Jacquet et al. 2021). However, the most important structural difference of the melt spherules compared to natural chondrules is the absence of glassy mesostasis, typical for natural porphyritic chondrules. This observation can likely be attributed to the use of pure forsterite as starting material, and consequently, future experiments could benefit from a mixture of olivine and plagioclase as initial material.

Furthermore, we observed one object, which resembles in a broad way compound chondrules since it consists of two partially molten spherules, and another one which seems to be the result of collisions of molten and initial material. However, their textures are not comparable to those of compound chondrules and incomplete melting is not typically observed in natural porphyritic chondrules. Some chondrules show evidence for repeated heating or chondrule recycling (e.g., Baecker et al. 2017; Krot et al. 2018; Schrader et al. 2018; Villeneuve et al. 2020; Jacquet et al. 2021), which would also contribute to complete instead of partial melting. Whether half-melted particles transformed with repeated melting to completely molten particles could not be determined in our experiment.

The limited spatial dimensions of the heating flash in our experiment caused only a very low amount of completely molten droplets, and consequently, the possibility of collisions between completely molten droplets was unlikely. Complete melting may have been prevented in many cases by the distance between a particle and the arc discharge or by large particle diameters. Due to the experimental conditions aboard the ISS, it was not possible to observe the distance between the particles and the arc discharge for specific samples. Future experiments could benefit from the usage of high-speed cameras.

In our experiment, the cooling rate of a single melt spherule is dominated by thermal radiation and is $>10^{5} \mathrm{~K} \mathrm{~h}^{-1}$ within the first seconds, which is in accordance with the cooling rates predicted for the nebular lightning hypothesis (Morris and Desch 2010). The cooling rate is of special interest in chondrule formation experiments because it is interpreted to be responsible for the formation of the different chondrule textures (detailed review in Jones et al. 2018). High 
cooling rates have been one of the counterarguments against the formation of chondrules by nebular lightning or other flash-heating formation scenarios (Hewins et al. 2000; Desch et al. 2012), since they are not in accordance with the thermal path of the most common chondrule texture type, porphyritic chondrules. Consequently, there are two possibilities for future microgravity experiments: either change the thermal history by employing a new technical approach or work with high cooling rates and change the sample material. The latter is supported by the idea that the cooling rate is not one of the decisive factors for the formation of different chondrule texture types.

For example, the cooling rate of spherules in our experiment is consistent with the results of experiments by Greenwood and Herbst (2021), who were able to reproduce porphyritic textures with chemical zoning with very high cooling rates. The forsterite crystals observed in our melt spherules formed by arc discharge aboard the ISS do not display chemical zoning since the starting material was pure forsterite, but this is an aspect that could be addressed in future experiments using more chemically complex starting material.

Another important parameter that may influence the chondrule formation is the interaction of melt with the surrounding gas (Tissandier et al. 2002; Libourel et al. 2006; Marrocchi and Chaussidon 2015; Villeneuve et al. 2015; Baecker et al. 2017; Marrocchi et al. 2018; Libourel and Portail 2018). The recent analytical work of Libourel and Portail (2018) and experiments from Villeneuve et al. (2015) proposed that the precursor material may have been heated well above the liquidus temperature and started crystallizing at high temperatures around $1800 \mathrm{~K}$ in reaction with the surrounding gas. The duration of the interaction lasted up to a few tens of minutes, with subsequent fast cooling at rates of up to $10^{6} \mathrm{~K} \mathrm{~h}^{-1}$ (Libourel and Portail 2018). They suggested that lightning and impact splash scenarios rather than shock waves seem better suited to realize the thermal history proposed in their model.

The melt spherules formed in the experiment also provide information about the incorporation of metal and gas-solid interactions during the experiment. There are different theories about the origin of $\mathrm{Fe}-\mathrm{Ni}$ inclusions in chondrules. Libourel and Portail (2018), for example, concluded that liquid metal droplets were incorporated into the chondrule melt during chondrule formation and served as nuclei for porphyritic textures, whereas other studies proposed that metal blebs are relicts of chondrule precursors (Marrocchi et al. 2019). The tungsten precipitation and inclusions observed in our melt spherules are due to tungsten evaporation from the electrodes prior to its interaction with the molten spherules. Although their incorporation is not directly comparable to the proposed origin of natural $\mathrm{Fe}-\mathrm{Ni}$ inclusions in chondrules, they demonstrate an interaction of the complete spherule with the surrounding $\mathrm{W}$-bearing gas at high temperatures during crystallization. Even though this period was much shorter (approximately several $100 \mu$ s) than the long duration experiments on gas-melt interactions (Villeneuve et al. 2015; Libourel and Portail 2018), it shows that interaction of gas and liquid is possible in this type of experiment and this topic could be studied with an improved experimental setup.

\section{CONCLUSION}

We carried out the first chondrule formation experiment under long-term microgravity conditions aboard the ISS. We have shown that freely floating $\mathrm{Mg}_{2} \mathrm{SiO}_{4}$ dust particles can be completely melted by arc discharges into melt spherules that can be considered to be chondrule analogs. Some microtextural properties, such as crystal sizes and orientations, morphologies, and metal inclusions, are very similar to those observed in natural chondrules. The cooling rates of the melt spherules in our experiments are much higher than the previously established cooling rates of the majority of chondrules, but consistent with recent results (e.g., Greenwood and Herbst 2021), which implies that the parameters of the experiment could be used for similar studies. The absence of mesostasis in the experimentally formed spherules can be attributed to the restricted initial composition, which was pure $\mathrm{Mg}_{2} \mathrm{SiO}_{4}$. This clearly prevents a more detailed comparison of our samples to natural chondrules.

Chondrule formation experiments under long-term microgravity conditions are still in a very early stage, but our experiments have provided novel experimental constraints for the formation of the first solids formed and processed in the early few million years of our solar system. As the feasibility of this experimental approach to study chondrule formation under long-term microgravity conditions is now established, more advanced experiments can be developed to study chemically more complex systems.

Acknowledgments-This project was realized with the help of A.A. Beck, O. Christ, P.-T. Genzel, and D. Mederos Leber. We thank the Hackerspace Ffm e. V., especially S. Fujita and J. Kerscher, R. Haseitl and S. Matthies for the help with the technical implementation and software development. We thank J. Weppler and the German Aerospace Center DLR for taking care of the organization of this project. We thank NanoRacks LLC and DreamUp for the payload opportunity. We 
also thank the astronauts aboard the ISS under the command of A. Gerst for the implementation of the experiment. We are grateful for financial support provided by the Dr. Rolf $M$. Schwiete Stiftung, the German Aerospace center DLR, NanoRacks LLC, DreamUp, Biovia, the BmWi (50JR1704) and DFG (BR2015/35-1; Wi1232/44-1), Nordlicht GmbH, and ZEISS. We acknowledge DESY (Hamburg, Germany), a member of the Helmholtz Association HGF, for the provision of experimental facilities. Parts of this research were carried out at PETRA III. BW is grateful for support through the BIOVIA Science Ambassador program. WOA Institution: Goethe-Universitat Frankfurt am Main, Blended DEAL: Projekt DEAL. Open Access funding enabled and organized by Projekt DEAL.

Data Availability Statement - Data available on request from the authors.

\section{Editorial Handling-Dr. Yves Marrocchi}

\section{REFERENCES}

Baecker B., Rubin A. E., and Wasson J. T. 2017. Secondary melting events in Semarkona chondrules revealed by compositional zoning in low-Ca pyroxene. Geochimica et Cosmochimica Acta 211:256-279.

Bland P. A., Howard L. E., Prior D. J., Wheeler J., Hough R. M., and Dyl K. A. 2011. Earliest rock fabric formed in the solar system preserved in a chondrule rim. Nature Geoscience 4:244-247.

Blander M., Planner H. N., Keil K., Nelson L. S., and Richardson N. L. 1976. The origin of chondrules: Experimental investigation of metastable liquids in the system $\mathrm{Mg}_{2} \mathrm{SiO}_{4}-\mathrm{SiO}_{2}$. Geochimica et Cosmochimica Acta 40:889-896.

Bollard J., Connelly J. N., Whitehouse M. J., Pringle E. A., Bonal L., Jørgensen J. K., Nordlund A., Moynier F., and Bizzarro M. 2017. Early formation of planetary building blocks inferred from $\mathrm{Pb}$ isotopic ages of chondrules. Science Advances 3:e1700407.

Boss A. P. 1996. A concise guide to chondrule formation models. In Chondrules and the protoplanetary disk, edited by Hewins R. H., Jones R. H., and Scott E. R. D. Cambridge, UK: Cambridge University Press. pp. 257263.

Bowen N. L. and Schairer J. F. 1925. The system MgO-FeO$\mathrm{SiO}_{2}$. American Journal of Science 29:151-217.

Chaumard N., Humayun M., Zanda B., and Hewins R. H. 2018. Cooling rates of type I chondrules from Renazzo: Implications for chondrule formation. Meteoritics \& Planetary Science 53:984-1005.

Ciesla F. J. and Hood L. L. 2002. The nebular shock wave model for chondrule formation: Shock processing in a particle-gas suspension. Icarus 158:281-293.

Connolly H. C. and Jones R. H. 2016. Chondrules: The canonical and noncanonical views. Journal of Geophysical Research: Planets 121:1885-1899.
Connolly H. C., Jones B. D., and Hewins R. H. 1998. The flash melting of chondrules: An experimental investigation into the melting history and physical nature of chondrule precursors. Geochimica et Cosmochimica Acta 62:27252735.

Desch S. J. and Cuzzi J. N. 2000. The generation of lightning in the solar nebula. Icarus 143:87-105.

Desch S. J., Morris M. A., Connolly H. C. Jr., and Boss A. P. 2012. The importance of experiments: Constraints on chondrule formation models. Meteoritics \& Planetary Science 47:1139-1156.

Dodd R. T. and Teleky L. S. 1967. Preferred orientation of olivine crystals in porphyritic chondrules. Icarus 6:407-416.

Gattacceca J., Bouvier A., Grossman J., Metzler K., and Uehara M. 2019. The Meteoritical Bulletin, No. 106. Meteoritics \& Planetary Science 54:469-471.

Greenwood R. C. and Herbst W. 2021. New constraints on chondrule formation from experimental reproduction of aluminum and titanium in chondrule olivine (abstract \#1617). 52nd Lunar and Planetary Science Conference. CD-ROM.

Güttler C., Poppe T., Wasson J. T., and Blum J. 2008. Exposing metal and silicate charges to electrical discharges: Did chondrules form by nebular lightning? Icarus 195:504-510.

Hewins R. H. and Fox G. E. 2004. Chondrule textures and precursor grain size: An experimental study. Geochimica et Cosmochimica Acta 68:917-926.

Hewins R. H., Zanda R. H., Horányi M., Robertson S., Den Hartog D. J., and Fiksel G. 2000. The trouble with flash heating (abstract \#1675). 31st Lunar and Planetary Science Conference. CD-ROM.

Hewins R. H., Connolly H. C. Jr., and Libourel G. 2005. Experimental constraints on chondrule formation. Chondrites and the Protoplanetary Disk 341:286-316.

Horányi M., Morfill G., Goertz C. K., and Levy E. H. 1995. Chondrule formation in lightning discharges. Icarus 114:174-185.

Hubbard A. and Ebel D. S. 2018. Evaluating non-shock, noncollisional models for chondrule formation. In Chondrules. Records of protoplanetary disk processes, edited by Russell S. S., Connolly H. C., and Krot A. N. Cambridge, UK: Cambridge University Press. pp. 400-427.

Jacquet E., Piralla M., Kersaho P., and Marrocchi Y. 2021. Origin of isolated olivine grains in carbonaceous chondrites. Meteoritics \& Planetary Science 56:13-33.

Johansen A. and Okuzumi S. 2018. Harvesting the decay energy of $26 \mathrm{Al}$ to drive lightning discharge in protoplanetary discs. Astronomy \& Astrophysics 609:A31.

Johansen A., Low M. M., Mac L. P., and Bizzarro M. 2015. Growth of asteroids, planetary embryos, and Kuiper belt objects by chondrule accretion. Science Advances 1:e1500109.

Jones R. H. 1990. Petrology and mineralogy of Type II, FeO-rich chondrules in Semarkona (LL3.0): Origin by closed-system fractional crystallization, with evidence for supercooling. Geochimica et Cosmochimica Acta 54:1785-1802.

Jones R. H., Grossman J., and Rubin A. 2005. Chemical, mineralogical and isotopic properties of chondrules: Clues to their origin. Chondrites and the Protoplanetary Disk 341:251.

Jones R. H., Villeneuve J., and Libourel G. 2018. Thermal histories of chondrules. In Chondrules; records of protoplanetary disk processes, edited by Russell S. S., Connolly H. C., and Krot A. N. Cambridge, UK: Cambridge University Press. pp. 57-90. 
Krot A. N., Amelin Y., Cassen P., and Meibom A. 2005. Young chondrules in $\mathrm{CB}$ chondrites from a giant impact in the early solar system. Nature 436:989-992.

Krot A. N., Nagashima K., Libourel G., and Miller K. E. 2018. Multiple mechanisms of transient heating events in the protoplanetary disk: Evidence from precursors of chondrules and igneous $\mathrm{Ca}$, Al-rich inclusions. In Chondrules: Records of protoplanetary disk processes, edited by Russell S. S., Connolly H. C., and Krot A. N. Cambridge, UK: Cambridge University Press. pp. 11-56.

Levy E. H. 1988. Energetics of chondrule formation. In Meteorites and the early solar system, edited by Kerridge $\mathbf{J}$. F. and Matthews M. S. Tucson, Arizona: University of Arizona Press. pp. 697-711.

Libourel G. and Portail M. 2018. Chondrules as direct thermochemical sensors of solar protoplanetary disk gas. Science Advances 4:eaar3321.

Libourel G., Krot A., and Tissandier L. 2006. Role of gasmelt interaction during chondrule formation. Earth and Planetary Science Letters 251:232-240.

Lichtenberg T., Golabek G. J., Dullemond C. P., Schönbächler M., Gerya T. V., and Meyer M. R. 2018. Impact splash chondrule formation during planetesimal recycling. Icarus 302:27-43.

Marrocchi Y. and Chaussidon M. 2015. A systematic for oxygen isotopic variation in meteoritic chondrules. Earth and Planetary Science Letters 430:308-315.

Marrocchi Y. and Libourel G. 2013. Sulfur and sulfides in chondrules. Geochimica et Cosmochimica Acta 119:117-136.

Marrocchi Y., Villeneuve J., Batanova V., Piani L., and Jacquet E. 2018. Oxygen isotopic diversity of chondrule precursors and the nebular origin of chondrules. Earth and Planetary Science Letters 496:132-141.

Marrocchi Y., Euverte R., Villeneuve J., Batanova V., Welsch B., Ferrière L., and Jacquet E. 2019. Formation of CV chondrules by recycling of amoeboid olivine aggregate-like precursors. Geochimica et Cosmochimica Acta 247:121-141.

McNally C., Hubbard A., Low M.-M., Ebel D., and D'Alessio P. 2013. Mineral processing by short circuits in protoplanetary disks. The Astrophysical Journal Letters 767:L2.

Mishra B., Manvar P., Choudhury K., Karagadde S., and Srivastava A. 2020. Experiments to understand crystallization of levitated high temperature silicate melt droplets under low vacuum conditions. Scientific Reports 10:20910.

Moosmann J., Ershov A., Weinhardt V., Baumbach T., Prasad M. S., LaBonne C., Xiao X., Kashef J., and Hofmann R. 2014. Time-lapse X-ray phase-contrast microtomography for in vivo imaging and analysis of morphogenesis. Nature Protocols 9:294-304.

Morlok A., Sutton Y. C., Braithwaite N. S. J., and Grady M. M. 2012. Chondrules born in plasma? Simulation of gasgrain interaction using plasma arcs with applications to chondrule and cosmic spherule formation. Meteoritics \& Planetary Science 47:2269-2280.

Morris M. A. and Boley A. C. 2018. Formation of chondrules by shock waves. In Chondrules: Records of protoplanetary disk processes, edited by Russell S. S., Connolly H. C., and Krot A. N. Cambridge, UK: Cambridge University Press. pp. 375-399.

Morris M. A. and Desch S. J. 2010. Thermal histories of chondrules in solar nebula shocks. The Astrophysical Journal 722:1474-1494.
National Institute of Standards and Technology (NIST) n.d. Solid Phase Heat Capacity (Shomate Equation). https:// webbook.nist.gov/cgi/cbook.cgi?ID $=$ C10034943\&Units $=$ SI\&Mask $=2 \&$ Type $=$ JANAFS \&Plot $=$ on\#JANAFS . Accessed June 06, 2020.

Ogurreck M., Wilde F., Herzen J., Beckmann F., Nazmov V., Mohr J., Haibel A., Müller M., and Schreyer A. 2013. The nanotomography endstation at the PETRA III Imaging Beamline. Journal of Physics: Conference Series 425:182002.

Piani L., Marrocchi Y., Libourel G., and Tissandier L. 2016. Magmatic sulfides in the porphyritic chondrules of EH enstatite chondrites. Geochimica et Cosmochimica Acta 195:84-99.

Poppe T., Güttler C., and Springborn T. 2015. Thermal metamorphoses of cosmic dust aggregates: Experiments by furnace, electrical gas discharge, and radiative heating. Earth, Planets and Space 62:53-56.

Radomsky P. M. and Hewins R. H. 1990. Formation conditions of pyroxene-olivine and magnesian olivine chondrules. Geochimica et Cosmochimica Acta 54:34753490 .

Rueden C. T., Schindelin J., Hiner M. C., DeZonia B. E., Walter A. E., Arena E. T., and Eliceiri K. W. 2017. Image J2: ImageJ for the next generation of scientific image data. BMC Bioinformatics 18:529.

Russell S. S., Connolly H. C., and Krot A. N., eds. 2018. Chondrules: Records of protoplanetary disk processes. Cambridge, UK: Cambridge University Press. pp. 450.

Sanders I. S. and Scott E. R. D. 2012. The origin of chondrules and chondrites: Debris from low-velocity impacts between molten planetesimals? Meteoritics \& Planetary Science 47:2170-2192.

Schrader D. L., Nagashima K., Waitukaitis S. R., Davidson J., McCoy T. J., Connolly H. C., and Lauretta D. S. 2018. The retention of dust in protoplanetary disks: Evidence from agglomeratic olivine chondrules from the outer solar system. Geochimica et Cosmochimica Acta 223:405-421.

Schrader D. L., Nagashima K., Davidson J., McCoy T. J., Ogliore R. C., and Fu R. R. 2020. Outward migration of chondrule fragments in the early solar system: O-isotopic evidence for rocky material crossing the Jupiter Gap? Geochimica et Cosmochimica Acta 282:133-155.

Spahr D., Koch T. E., Merges D., Beck A. A., Bohlender B., Carlsson J. M., Christ O., Fujita S., Genzel P.-T., Kerscher J., Knautz T., Lindner M., Mederos Leber D., Milman V., Morgenroth W., Wilde F., Brenker F. E., and Winkler B. 2020. A chondrule formation experiment aboard the ISS: Experimental set-up and test experiments. Icarus 350:113898.

Tenner T. J., Nakashima D., Ushikubo T., Kita N. T., and Weisberg M. K. 2015. Oxygen isotope ratios of FeO-poor chondrules in CR3 chondrites: Influence of dust enrichment and $\mathrm{H} 2 \mathrm{O}$ during chondrule formation. Geochimica et Cosmochimica Acta 148:228-250.

Tenner T. J., Ushikubo T., Kurahashi E., Kita N. T., and Nagahara H. 2013. Oxygen isotope systematics of chondrule phenocrysts from the CO3.0 chondrite Yamato 81020: Evidence for two distinct oxygen isotope reservoirs. Geochimica et Cosmochimica Acta 102:226-245.

Tissandier L., Libourel G., and Robert F. 2002. Gas-melt interaction and their bearing on chondrule formation. Meteoritics \& Planetary Science 37:1377-1389. 
Tkalcec B. J., Golabek G. J., and Brenker F. E. 2013. Solidstate plastic deformation in the dynamic interior of a differentiated asteroid. Nature Geoscience 6:93-97.

Ushikubo T., Nakashima D., Kimura M., Tenner T. J., and Kita N. T. 2013. Contemporaneous formation of chondrules in distinct oxygen isotope reservoirs. Geochimica et Cosmochimica Acta 109:280-295.

Villeneuve J., Libourel G., and Soulié C. 2015. Relationships between type I and type II chondrules: Implications on chondrule formation processes. Geochimica et Cosmochimica Acta 160:277-305.

Villeneuve J., Marrocchi Y., and Jacquet E. 2020. Silicon isotopic compositions of chondrule silicates in carbonaceous chondrites and the formation of primordial solids in the accretion disk. Earth and Planetary Science Letters 542:116318.

Wdowiak T. J. 1983. Experimental investigation of electrical discharge formation of chondrules. In Chondrules and their origins, edited by King E. A. Houston, Texas: Lunar and Planetary Institute. pp. 279-283.

Wilde W., Ogurreck M., Greving I., Hammel J. U., Beckmann F., Hipp A., Lottermoser L., Khokhriakov I., Lytaev P., Dose T., Burmester H., Müller M., and Schreyer A. 2016. Micro-CT at the imaging beamline P05 at PETRA III. AIP Conference Proceedings 1741: \#30035. 